\title{
ON AN EMISSION OF NEGATIVELY CHARGED PARTICLES PRODUCED BY CANAL RAYS. ${ }^{1}$
}

By L. W. Austin.

THE work described in the following paper was undertaken for the purpose of studying the possible secondary radiations produced by the impact of the canal rays on a metal. Since it is known that the canal rays consist of positively charged particles of molecular dimensions travelling with a velocity sometimes exceeding $10^{8} \mathrm{~cm} . / \mathrm{sec}$, ${ }^{2}$ it seemed probable that they might give rise to reflected positive rays analogous to the reflected cathode rays studied by several physicists, ${ }^{3}$ and perhaps also to an emission of negative particles.

The tube used in the work is shown in Fig. I. It was divided

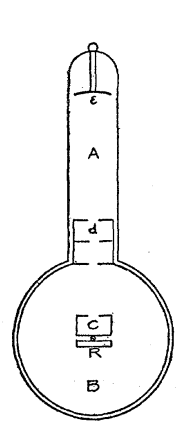

Fig. :. into two parts $A$ and $B$. The part $A$ was $7 \mathrm{~cm}$. long and $2.5 \mathrm{~cm}$. in diameter. $B$ was $7 \mathrm{~cm}$. in diameter and was separated from $A$ by the system of brass diaphragms $d$ with openings $2 \mathrm{~mm}$. in diameter. The aluminum electrode $e$ formed the anode while the front of the diaphragms served as cathode. The canal rays passed backward through the openings in the diaphragms into the observation space $B$, which was lined with fine brass netting. This with the diaphragms was connected to earth, thus forming an enclosed space practically free from the direct influence of the discharge. In looking for the reflection of the canal rays the following arrangement was used. A small brass cylinder $C, 1.5 \mathrm{~cm}$. in diameter and $\mathrm{I} \mathrm{cm}$. long, closed except for an opening $4 \mathrm{~mm}$. in

1 Read before the Washington Philosophical Society, February 10, 1906.

2 W. Wien, Wied. Ann., Vol. 65, p. 440, 1898; Ewers, Wied. Ann., Vol. 69, p. 167, 1899; Austin, Bulletin of Bureau of Standards, Vol. I, p. 439, I905.

3 Goldstein, Wied. Ann., Vol. 15, p. 254, I882 ; Campbell Swinton, Proc. Roy. Soc., Vol. 64, p. 377, 1899; Starke, Wied. Ann., Vol. 66, p. 49, 1898; Ann. d. Phys., Vol. 3, p. 75, I900; Austin and Starke, Ann. d. Phys., Vol. 19, p. 27 I, 1902. 
diameter in the front, was fastened to a brass spindle. To the other side of the spindle was attached a brass disk $R, 1.5 \mathrm{~cm}$. in diameter. The spindle passed up through a ground glass joint and was connected to earth through a D'Arsonval galvanometer giving one division for $2.3 \times 10^{-9} \mathrm{amp}$. This was shunted so as to have a lower sensibility during most of the work. The spindle could be turned so that the canal rays could be made to enter the cylinder $C$ or strike the reflector $R$. In case the rays entered the cylinder all of the positive charges conveyed by them were caught and conducted to earth through the galvanometer. When the reflecting disk $R$ was turned toward the rays the reflected portion would rebound and only the charges carried by the absorbed portion would pass through the galvanometer to earth. The difference in the currents indicated by the galvanometer in the two cases would represent the reflected portion of the rays.

The current for the tube was supplied by a motor-driven electrical machine with four moving plates capable of generating about 0.0003 amp. when driven at full speed. The amount of current could be varied at will by changing the speed of the machine. The difference of potential between the electrodes of the tube was measured by means of a Braun electrometer having a range of 500 to I0,000 volts. The vacuum was produced by a motor-driven Geryk oil pump.

The first observations showed that the reflection of the canal rays was entirely masked by a secondary negative emission, for the reflector current was found to be larger than the cylinder current.

As the work progressed it became evident that there were irregularities in the phenomenon of a very puzzling character. It was found that from time to time the ratio of reflector current to cylinder current varied between rather wide limits, and that these variations did not depend as far as could be observed either on the amount of current flowing or on the degree of vacuum and difference in potential between the electrodes. These irregularities have made it impossible to carry out the work with the same quantitative exactness as in the case of the cathode rays.

Irregularities have also been observed by $\mathrm{Wien}^{1}$ in his determi1 W. Wien, loc. cit. 
nation of the ratio $e / m$ for the canal rays, and it seems possible that they may arise in both cases at least in part from common causes. As an explanation of Wien's results, J. J. Thomson has supposed that perhaps some of the positive particles have their charges neutralized by taking on negative electrons as they pass through the gas, and that they again become ionized when they strike against a solid and are thus able to produce fluorescence. If we suppose that such neutral particles are also capable of producing the negative emission we have a possible explanation of our irregularities, for the presence of these neutral particles could not in any way be discovered in the cylinder while they would produce a difference in the reflector current. Another difficulty in quantitative measurements with canal rays lies in their great absorption in thin layers of foreign substances on the surfaces struck by them. ${ }^{1}$ Notwithstanding these difficulties observations made on the same day and without introducing air into the tube generally agreed within a few per cent.

If we call the galvanometer current from the cylinder $C$ and from the reflector $R,(R-C) / C$ will represent the ratio between the emitted negative particles minus the reflected positive particles and the positive particles producing them. In Table I. are given the mean values of series taken on three different days. Observations were

TABLE I.

\begin{tabular}{c|c|c|c}
\hline \multirow{2}{*}{ Discharge P. D. Volts. } & \multicolumn{2}{|c|}{ Galvanometer Deflections. } & 10-7 amp. \\
\cline { 2 - 3 } & $C$ & $R$ & $\frac{R-C^{\prime}}{C}$ \\
\hline 7,000 & 78 & 114 & 0.46 \\
7000 & 102 & 146 & .43 \\
6,000 & 77 & 109 & .42 \\
\hline
\end{tabular}

1 In this connection the following experiment was carried out: Two brass reflectors were carefully polished and cleaned with alcohol and then fastened to the two sides of the spindle in the tube. When struck by the canal rays both gave almost identical galvanometer deflections. One of the reflectors was then removed and rubbed over with the thinnest possible film of pump grease. When the reflectors were again compared the greased one gave a galvanometer deflection of only $0.5 \mathrm{~cm}$., while the bright one gave 18 $\mathrm{cm}$. When the machine was reversed so that cathode rays struck the reflectors it was found that the grease film was almost without effect and that the two deflections were practically identical. Even after rubbing the greased reflector as clean as possible with a dry flannel cloth, it still gave only about half the full deflection for the canal rays. 
also made on other reflectors than brass. [ $\mathrm{Cu}$ gave practically the same results, $\mathrm{Pt}$ a larger relative negative emission and zinc a smaller one. But as observations with external E.M.F. in the galvanometer circuit showed that the conductivity in the space exposed to the canal rays was very high, so that a difference of potential of one volt between the reflector and earth produced a deflection amounting to a large fraction of the total effect due to the caanl rays, it was not thought best to put too much weight on these observations in which different metals were immersed in the conducting gas. For in this case it seems possible that the two metals would develop differences of potential as when immersed in an electrolyte. ${ }^{1}$

Since it is known that the negative emission produced when cathode rays strike a metal increases rapidly as the angle of incidence increases, ${ }^{2}$ observations were made to determine whether this was also true in the present case. As the spindle bearing the reflector could be rotated it was possible to cause the canal rays to strike at any desired angle.

Table II. shows a set of these observations. It is seen that the galvanometer current increases rapidly as the angle of incidence increases, being more than 40 per cent. greater at $70^{\circ}$ than at $0^{\circ}$.

TABLE II.

$\begin{array}{cc}\text { Angle of Incidence. } & \text { Galvanometer Deflections. }{ }^{10^{-7}} \text { amp. } \\ 0^{\circ} & 156 \\ 40 & 170 \\ 60^{\circ} & 196 \\ 70 & 223\end{array}$

And if we assume that the increase is entirely in the emission the difference amounts to about one and one half times.

In order to still further establish the existence of the negative emission and observe it directly the following arrangement was adopted: A cyclinder was constructed with the base insulated from the remaining portion by a hard rubber ring as shown in Fig. 2. Either part could be connected to earth through the galvanometer while the other was earthed directly. Any negative particles sent

${ }^{1}$ S. Arrhenius, Wied Ann., V'ol. 33, p. 638, 1888.

2 Austin and Starke, loc. cit. 
out from the base when struck by the canal rays were caught by the part $a$ and could be detected by being led to earth through the galvanometer. When the experiment was performed it was found as was expected that while the base $b$ gave a positive deflection as before, the part of the cylinder $a$ showed a negative current. Table III. gives the mean value of three sets of observations taken on

Table III. (See Fig. 2.)

\begin{tabular}{c|c|c|c}
\hline \multirow{2}{*}{ Discharge P. D. Volts. } & \multicolumn{2}{|c|}{ Galvanometer Deflections. I0 $^{-7}$ amp. } & $\frac{a}{b-a}$ \\
\cline { 2 - 3 } & $b$ & $a$ & \\
\hline 4,000 & +6.8 & -1.9 & 0.38 \\
“ & 7.5 & 2.2 & .41 \\
& 18.0 & 5.6 & .42 \\
\hline
\end{tabular}

different days. The ratio of negative emission minus reflected positive particles to canal rays is now expressed by $a_{j}^{\prime}(b-a)$ and the agreement with the results of Table I. is good.

Besides the Volta effect already mentioned there were two other possible spurious causes of the phenomenon, namely the presence of cathode rays mixed with the canal rays due to oscillations in the circuit and the action of ultra-violet light coming through the diaphragms from the discharge portion of the tube. As a magnetic field in the observation space strong enough to divert any cathode rays from the reflector had no effect on the emission, and as all action in the observation space ceased when the cathode rays in the discharge tube were diverted so that the canal rays did not pass the diaphragms these possible sources of error were considered eliminated.

The velocity of the new emission seemed to be a question of considerable interest, and accordingly attempts were made to determine it approximately by the method of magnetic deflection. In the first experiment a brass plate $p, \mathrm{I} .5 \mathrm{~cm}$. long and $\mathrm{I} \mathrm{cm}$. wide, insulated from the spindle was placed $\mathrm{I} \mathrm{cm}$. above the center of the reflector $R$ as shown in Fig. 3. $R$ was connected directly to earth and $p$ to earth through the galvanometer. A second experiment was arranged as in Fig. 4. Here the plate $p$ was parallel to 
the reflector and separated from it by an earthed brass screen $S$. The magnetic field was produced by a horseshoe magnet and of course was not at all uniform. The strength of the magnet may be estimated from the observation that when brought within $3.5 \mathrm{~cm}$.

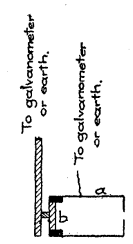

Fig. 2.

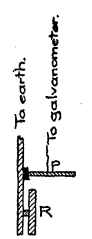

Fig. 3.

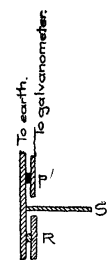

Fig. 4.

of the axis of the discharge portion of the tube the cathode rays at a potential of 4,000 volts were bent on a radius of about $4 \mathrm{~cm}$. The results of the two experiments are shown in Tables IV. and V.

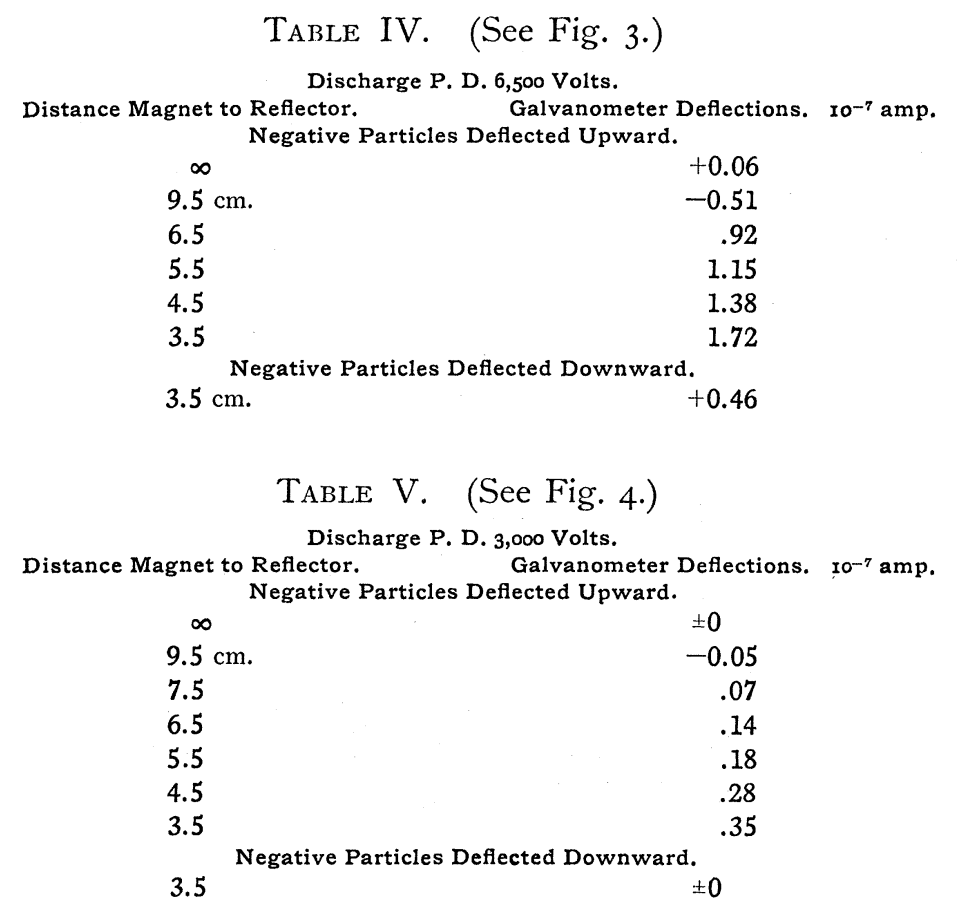


These observations show three things: $(\mathrm{I})^{1}$ That without magnetic field the negative emission strikes the plate $p$, Fig. 3 , and hence is not confined to a direction at right angles to the reflector and is diffuse. (2) When the negative particles are deflected away from $p$, Fig. 3, the deflection of the galvanometer is positive showing that there is a slight diffuse reflection of the canal rays themselves. (3) Both tables show that even with very weak fields tending to deflect the negative particles toward the plates the negative current is increased, but even the strongest field applied is insufficient to bring the negative current to a maximum. This indicates that there are particles of widely varying velocities in the emission some extremely slow and some whose velocities can not be much less than $0.2 \times 10^{10}$ $\mathrm{cm} . / \mathrm{sec}$. and may be very much greater.

Regarding the mechanism of the secondary radiation it is difficult to say anything with certainty. It is clear that, as the radiation takes place in a space free from any differences of potential, all parts being connected to earth, the energy not only for the separation of the negative electrodes but also for their velocity must come from the canal rays themselves unless we are willing to assume that their impact on the metal renders its atoms radioactive so that the electrons are expelled by some process of explosion. The fact that the emission of the electrons is greater at larger angles of incidence is also difficult of explanation especially in this case when the canal rays do not penetrate the metal surface and the explanation suggested by J. J. Thomson ${ }^{2}$ for the increased emission at large angles due to cathode rays cannot apply.

We have in the emission of negative electrons by a bombardment of positive particles a condition of things very similar to that involved in the passage of negative electricity into the gas at the cathode in the ordinary vacuum discharge, as in that case also it is generally believed that the negative particles are liberated by the impact of positive particles upon the cathode. If indeed the mechanism of the two cases is the same the present work indicates means of studying the nature of this portion of the discharge in vacuum under simplified conditions.

${ }^{1}$ Without magnetic field the deflections in the first experiment were sometimes positive and sometimes negative.

${ }^{2}$ J. J. Thomson, Conduction of Electricity through Gases, p. 509. 


\section{SUMMARY.}

I. When canal rays strike an earthed metal surface there is a weak diffuse' reflection of these rays.

2. There is also a diffuse emission of negative particles having widely varying velocities.

3. This negative emission increases as the angle of incidence of the canal rays increases and is probably similar in character to the secondary negative emission produced by cathode rays. ${ }^{1}$

National Bureau of Standards, Washington.

${ }^{1}$ Since the above was written Professor J. J. 'Thomson's article : Some Experiments on Kanal-Strahlen, Proc. Camb. Phil. Soc., Vol. 13, Part 4, p. 212, 1906, has appeared. 